\title{
Isolated Aseptic Powder Filling Method
}

National Cancer Institute

\section{Source}

National Cancer Institute. Isolated Aseptic Powder Filling Method. NCI Thesaurus. Code C112987.

A process that fills a dosage unit with a powder, which is performed in a closed environment under sterile conditions and without human intervention. 